\title{
PARITY OF RANKS OF ELLIPTIC CURVES WITH EQUIVALENT MOD $p$ GALOIS REPRESENTATIONS
}

\author{
SUDHANSHU SHEKHAR
}

(Communicated by Romyar T. Sharifi)

\begin{abstract}
Given two elliptic curves $E_{1}$ and $E_{2}$ defined over the field of rational numbers $\mathbb{Q}$ that have good and ordinary reduction at an odd prime $p$, and have equivalent, irreducible $\bmod p$ Galois representations, we study the variation of the parity of Selmer ranks and analytic ranks of $E_{1}$ and $E_{2}$ over certain number fields.
\end{abstract}

\section{INTRODUCTION}

Let $K$ be a number field. Fix an odd prime $p$ and let $\bar{K}$ denote a fixed algebraic closure of $K$. For an algebraic field extension $L / K$ and an elliptic curve $E$ defined over $K$, the $p$-Selmer group $\operatorname{Sel}_{p}(E / L)$ of $E$ over $L$ is defined by the exact sequence

$$
0 \longrightarrow \operatorname{Sel}_{p}(E / L) \longrightarrow H^{1}\left(L, E\left[p^{\infty}\right]\right) \stackrel{\oplus_{w} \delta_{w}}{\longrightarrow} \prod_{w} H^{1}\left(L_{w}, E\left(\overline{L_{w}}\right)\right) .
$$

Here, $w$ varies over the set of primes of $L, \overline{L_{w}}$ denotes a fixed algebraic closure of $L_{w}, E\left(\overline{L_{w}}\right)$ denotes the $\overline{L_{w}}$ points of $E, E\left[p^{\infty}\right]$ denotes the $p$-power torsion points of $E$ defined over $\bar{K}$ and $\oplus \delta_{w}$ denotes the natural restriction map.

Let $E_{1}$ and $E_{2}$ be two elliptic curves defined over the field of rational numbers $\mathbb{Q}$. We assume that $E_{1}$ and $E_{2}$ have good and ordinary reduction at the prime $p$. Let $K$ be a number field containing the $p$-th roots of unity. For an abelian group $M$, let $M\left[p^{n}\right]$ denote the $p^{n}$ torsion subgroup of $M$. We say that $E_{1}$ and $E_{2}$ are congruent at the prime $p$ if $E_{1}[p] \cong E_{2}[p]$ as modules over the absolute Galois group of $\mathbb{Q}$. The aim of this article is to study the variation of the parity of the $p$-Selmer ranks and analytic ranks of $E_{1}$ and $E_{2}$ over $K$.

Let $N_{i}$ denote the conductor of $E_{i}$ over $K$ and $\bar{N}_{i}$ denote the prime to $p$ conductor of the Galois module $E_{i}[p]$ over $K$ for $i=1,2$. Let $\Sigma$ be the finite set of primes of $K$ containing the primes of bad reduction of $E_{1}$ and $E_{2}$, the infinite primes and the primes above $p$. Put

$$
\Sigma_{0}:=\left\{v \in \Sigma|v| N_{1} / \bar{N}_{1} \text { or } v \mid N_{2} / \bar{N}_{2}\right\} .
$$

Let $S_{E_{i}}$ denote the set of primes $v \in \Sigma_{0}$ such that $E_{i}$ has split multiplicative reduction at $v$. Let $s_{p}\left(E_{i} / K\right)$ denote the rank of the Pontryagin dual of $\operatorname{Sel}_{p}\left(E_{i} / K\right)$, for $i=1,2$. Then we have the following result.

Received by the editors June 9, 2015 and, in revised form, August 21, 2015 and September 23, 2015 .

2010 Mathematics Subject Classification. Primary 14H52, 11F33, 11R23.

Key words and phrases. Iwasawa theory, congruences, Selmer groups, elliptic curves. 
Theorem 1.1. Let $E_{1}$ and $E_{2}$ be two elliptic curves defined over the field of rational numbers $\mathbb{Q}$ with good and ordinary reduction at the prime $p$. We further assume that the following holds:

(a) $E_{1}[p]$ is an irreducible $\operatorname{Gal}(\bar{K} / K)$-module.

(b) $K=K\left(\mu_{p}\right)$ where $\mu_{p}$ denotes the group of the $p$-th root of unity. $K$.

(c) $\operatorname{Sel}_{p}\left(E_{1} / K_{c y c}\right)[p]$ is finite where $K_{c y c}$ denotes the cyclotomic $\mathbb{Z}_{p}$-extension of

If $E_{1}$ and $E_{2}$ are congruent at the prime $p$, then we have that

$$
s_{p}\left(E_{1} / K\right)+\left|S_{E_{1}}\right| \equiv s_{p}\left(E_{2} / K\right)+\left|S_{E_{2}}\right| \bmod 2 .
$$

Remark 1.2. (i) If $E_{1}$ and $E_{2}$ are congruent at the prime $p$, then the assumption (a) holds for $E_{1}$ if and only if the same holds for $E_{2}$.

(ii) The assumption $(c)$ holds for $E_{1}$ if and only if it holds for $E_{2}$ (see Gr1, cf. Page 237] or Theorem 2.2 below).

We also mention that the assumption $(c)$ holds for $E_{1}$ if and only if the cyclotomic Iwasawa $\mu$-invariant of $E_{1}$ vanishes. In fact, there is no example known for which the assumption $(a)$ holds but assumption $(c)$ does not hold. There is a conjecture by Greenberg which asserts that if $E$ is an elliptic curve defined over $\mathbb{Q}$ with good ordinary reduction at $p$, then there exists a $\mathbb{Q}$-isogenous elliptic curve $E^{\prime}$ whose cyclotomic Iwasawa $\mu$-invariant vanishes ([Gr, Conjecture 1.11]). In particular, if $E[p]$ is an irreducible $\operatorname{Gal}(\overline{\mathbb{Q}} / \mathbb{Q})$-module, then the cyclotomic $\mu$-invariant of $E$ should vanish. This conjecture is false over a general number field. In his thesis, Drinen found examples of elliptic curves $E$ defined over $\mathbb{Q}$ with good ordinary reduction at $p$ and number fields $K$ such that every member of the $K$-isogeny class of $E$ has the positive cyclotomic $\mu$-invariant (see [Dr, Section 4] or [Dr1]). The examples found in $\overline{\mathrm{Dr}}$ do not satisfy the assumption $(a)$. We refer the reader to Remarks 3.4 and 3.7 and Section 4 for a discussion on the assumption $(b)$.

It is well known that the rank of an elliptic curve $E$ defined over a number field $K$ is determined by the $\operatorname{Gal}(\bar{K} / K)$-module structure of $E\left[p^{\infty}\right]$. This is a consequence of the Tate conjecture for elliptic curves proved by Faltings (see [Gr1, Page 232]). Although the Galois module structure of $E[p]$ does not determine the rank of $E$ over $K$, motivated by the conjecture of Tate, Greenberg and Vatsal (GV]) studied the variation of various algebraic, arithmetic and analytic invariants associated to elliptic curves under congruence. Specifically, they considered the cyclotomic algebraic and analytic Iwasawa invariants associated to elliptic curves defined over $\mathbb{Q}$ and provided a precise formula for the difference of the Iwasawa $\lambda$-invariants of two congruent elliptic curves. The proof of Theorem 1.1 crucially relies on the methods and results of Greenberg and Vatsal. We use recent results on the special cases of the parity conjecture (see Theorem 4.3 below) and Theorem 1.1 to compare the analytic rank of congruent elliptic curves.

It is an interesting problem to prove a relation analogous to (3) for the analytic rank of congruent elliptic curves independent of Theorem 1.1. Moreover, this may help in producing new examples of elliptic curves for which $p$-parity conjecture (see Conjecture 1) is true. Given an elliptic curve $E$ defined over $\mathbb{Q}$, Rubin and Silverberg (see $[\overrightarrow{\mathrm{RS}}]$ ) produced certain families of infinitely many elliptic curves which are congruent to $E$ at $p=3$ and 5 . In Section 4 of this article, we further compute the parity of $p$-Selmer ranks and analytic ranks of elliptic curves in such families over certain number fields using Theorem 1.1 
To prove our result, we shall need an equivalent but different definition of the Selmer groups over $K_{c y c}$ of elliptic curves with good and ordinary reduction at $p$. In Section 2, we recall this equivalent definition which is different from the definition (1) of the Selmer groups of elliptic curves defined over $K$. We also recall the definition of the Selmer group of the residual Galois representation of an elliptic curve at $p$. In Section 3 , we compare the parity of the $\lambda$-invariants of $E_{1}$ and $E_{2}$. Using this comparison we prove our main result which compares the parity of the Selmer ranks and the analytic ranks of $E_{1}$ and $E_{2}$ in Section 4 . To compare the parity of the $\lambda$-invariants of $E_{1}$ and $E_{2}$ we need to use the results of Greenberg in Gr1]. Further, we use the results of Hachimori and Matsuno which describe the $p$-power torsion points of an elliptic curve over the cyclotomic $\mathbb{Z}_{p}$-extension of a non-archimedean local field (see [HM]). In Section 4, we discuss several examples of elliptic curves to illustrate applications of our result and also to explain the assumptions in Theorem 1.1

\section{Selmer Group}

Let $E$ be an elliptic curve defined over the number field $K$ and $N_{K}$ be the conductor of $E$ over $K$. Suppose that $E$ has good and ordinary reduction at the primes above $p$ and that $E[p]$ is an irreducible $\operatorname{Gal}(\bar{K} / K)$-module. Let $\Sigma$ be a finite set of primes of $K$ containing the primes above $p$, the infinite primes and primes of bad reduction of $E$. Let $K_{\Sigma}$ denote the maximal algebraic extension of $K$ unramified outside $\Sigma$.

Let $K_{c y c}$ denote the cyclotomic $\mathbb{Z}_{p}$ extension of $K$ and $\Gamma:=\operatorname{Gal}\left(K_{c y c} / K\right)$. Put $\Lambda:=\lim _{\leftarrow} \mathbb{Z}_{p}\left[\Gamma / \Gamma^{p^{n}}\right] \cong \mathbb{Z}_{p}[[X]]$, where the inverse limit is taken over the natural projection maps.

Let $\widetilde{E}_{v}$ denote the reduction of the elliptic curve $E$ at the prime $v \mid p$. For an abelian group $M$, put $M\left[p^{\infty}\right]:=\bigcup_{n} M\left[p^{n}\right]$. Since $E$ has good ordinary reduction at the primes dividing $p$, we have that the $\operatorname{Gal}\left(\bar{K}_{v} / K_{v}\right)$-module $\widetilde{E}_{v}\left[p^{\infty}\right]$ is unramified for every prime $v \mid p$. For a Galois extension $L \subset K_{\Sigma}$ of $K$ and a prime $v \nmid p$ of $K$, put

$$
\mathcal{H}_{v}(L, E):=\prod_{w \mid v} H^{1}\left(L_{w}, E\left[p^{\infty}\right]\right)
$$

If $v \mid p$, then put

$$
\mathcal{H}_{v}(L, E):=\prod_{w \mid v} H^{1}\left(I_{w}, \widetilde{E}_{v}\left[p^{\infty}\right]\right)
$$

where $I_{w}$ denotes the inertia subgroup of $\operatorname{Gal}\left(\overline{L_{w}} / L_{w}\right)$. Let $\Sigma_{0}$ be a subset of $\Sigma$ not containing the primes above $p$ and the infinite primes. The imprimitive $\Sigma_{0}$-Selmer group $\operatorname{Sel}_{p}^{\Sigma_{0}}(E / L)$ of $E$ over $L$ is defined by the following exact sequence:

$$
0 \longrightarrow \operatorname{Sel}_{p}^{\Sigma_{0}}(E / L) \longrightarrow H^{1}\left(K_{\Sigma} / L, E\left[p^{\infty}\right]\right) \longrightarrow \prod_{v \in \Sigma \backslash \Sigma_{0}} \mathcal{H}_{v}(L, E)
$$

When $\Sigma_{0}$ is empty, we drop it from the notation $\operatorname{Sel}_{p}^{\Sigma_{0}}(E / L)$ and write $\operatorname{Sel}_{p}(E / L)$ (see [Gr1, section 1] and GV, Page 42] for the equivalence of definitions (11) and (44). We next define the Selmer group of the Galois module $E[p]$. For a prime $v \nmid p$ of $K$, put

$$
\mathcal{H}_{v}(L, E[p]):=\prod_{w \mid v} H^{1}\left(L_{w}, E[p]\right) .
$$


If $v \mid p$, then put

$$
\mathcal{H}_{v}(L, E[p]):=\prod_{w \mid v} H^{1}\left(I_{w}, \widetilde{E}_{v}[p]\right) .
$$

The imprimitive $\Sigma_{0}$-Selmer group $\operatorname{Sel}_{p}^{\Sigma_{0}}(E[p] / L)$ of $E[p]$ over $L$ is defined by the following exact sequence:

$$
0 \longrightarrow \operatorname{Sel}_{p}^{\Sigma_{0}}(E[p] / L) \longrightarrow H^{1}\left(K_{\Sigma} / L, E[p]\right) \longrightarrow \prod_{v \in \Sigma \backslash \Sigma_{0}} \mathcal{H}_{v}(L, E[p]) .
$$

Let $\bar{N}_{K}$ denote the prime to $p$-conductor of the Galois module $E[p]$. For a finite prime $w$ of $K_{c y c}$ lying above a finite prime $v \nmid p$ of $K$, we consider the natural homomorphism

$$
\gamma_{w}: H^{1}\left(K_{c y c, w}, E[p]\right) \longrightarrow H^{1}\left(K_{c y c, w}, E\left[p^{\infty}\right]\right)[p]
$$

and for a prime $w \mid v$ of $K_{c y c}$ such that $v \mid p$ consider the map

$$
\gamma_{w}: H^{1}\left(I_{w}, \tilde{E}_{v}[p]\right) \longrightarrow H^{1}\left(I_{w}, \tilde{E}_{v}\left[p^{\infty}\right]\right)[p] .
$$

Using [EPW, Lemma 4.1.2] the next lemma can be proved as in [SS, Theorem 3.4]. We reproduce the proof for the convenience of the reader.

Lemma 2.1. Let $v \nmid N_{K} / \bar{N}_{K}$ be a finite prime of $K$. Then, $\gamma_{w}$ is an injective homomorphism for every prime $w \mid v$ of $K_{\text {cyc }}$.

Proof. First suppose that $w \mid p$. In this case the kernel of $\gamma_{w}$ is equal to $H^{0}\left(I_{w}, \tilde{E}_{p^{\infty}}\right) / p$. Since $\tilde{E}_{p^{\infty}}$ is divisible and unramified, the kernel of $\gamma_{w}=0$. Now consider a prime $w \nmid p N_{K} / \bar{N}_{K}$. Then by an argument similar to EPW, Lemma 4.1.2], we get that $H^{0}\left(K_{c y c, w}, E\left[p^{\infty}\right]\right)$ is divisible. Therefore, the kernel $H^{0}\left(K_{c y c, w}, E\left[p^{\infty}\right]\right) / p$ of $\gamma_{w}$ is trivial.

As in Section 1 we consider two elliptic curves $E_{1}$ and $E_{2}$ defined over $\mathbb{Q}$ and state the following assumptions for further reference in the article.

Assumption 1. (i) $E_{1}$ (resp. $E_{2}$ ) has good and ordinary reduction at $p$.

(ii) $E_{1}[p]$ is an irreducible $\operatorname{Gal}(\bar{K} / K)$-module.

(iii) $K=K\left(\mu_{p}\right)$.

(iv) $\operatorname{Sel}_{p}\left(E_{1} / K_{c y c}\right)[p]$ is a finite group where $K_{\text {cyc }}$ denote the cyclotomic $\mathbb{Z}_{p^{-}}$ extension of $K$.

If Assumption 1(iv) holds, then the Pontryagin dual $X\left(E / K_{c y c}\right)$ of $\operatorname{Sel}_{p}\left(E / K_{c y c}\right)$ is a finitely generated $\mathbb{Z}_{p}$-module. Now suppose that the Assumption 1(ii) holds. Then $E(K)[p]=0$ and therefore [Gr, Proposition 4.14] implies that $\operatorname{Sel}_{p}\left(E / K_{c y c}\right)$ is divisible. We mention that [Gr, Proposition 4.14] is stated only when $\Sigma_{0}$ is the empty set. To prove the general case, consider the exact sequence

$$
0 \longrightarrow \operatorname{Sel}\left(E / K_{c y c}\right) \longrightarrow \operatorname{Sel}^{\Sigma_{0}}\left(E / K_{c y c}\right) \stackrel{\psi}{\longrightarrow} \prod_{v \in \Sigma_{0}} H_{v}\left(K_{c y c}, E\right) \longrightarrow 0 .
$$

The surjectivity of $\psi$ is a consequence of the Assumption 1)(iv) which implies that $\operatorname{Sel}\left(E / K_{\text {cyc }}\right)$ is a torsion $\Lambda$-module (cf. GV, Proposition 2.1, Corollary 2.3]; see also [HV] Theorem 7.2]). Now, it is well known that $H_{v}\left(K_{c y c}, E\right)$ is a divisible group for every finite prime $v$ of $K$ (see [Gr, Lemma 4.5 and successive paragraph]). Hence, $\operatorname{Sel}^{\Sigma_{0}}\left(E / K_{c y c}\right)$ is a divisible group. From [Gr2, Proposition 2] and using the fact 
that there are finitely many primes of $K_{c y c}$ lying above a finite prime of $K$, we see that the Pontryagin dual of $\mathcal{H}_{v}\left(K_{c y c}, E\right)$ is a finitely generated $\mathbb{Z}_{p}$-module. Put

$$
\lambda_{E}^{\Sigma_{0}}:=\operatorname{rank}_{\mathbb{Z}_{p}} X^{\Sigma_{0}}\left(E / K_{c y c}\right) .
$$

Since $\operatorname{Sel}_{p}^{\Sigma_{0}}\left(E / K_{c y c}\right)$ is divisible, $X^{\Sigma_{0}}\left(E / K_{c y c}\right)$ is $p$-torsion free and

$$
\lambda_{E}^{\Sigma_{0}}=\operatorname{rank}_{\mathbb{Z}_{p} / p} X^{\Sigma_{0}}\left(E / K_{c y c}\right) / p .
$$

Next we compare the Selmer group of an elliptic curve $E$ with the Selmer group of $E[p]$ over $K$ under Assumption [1. For this we use the method of Greenberg (Gr1]) and Greenberg-Vatsal ([GV]). Greenberg and Vatsal considered the case $K=\mathbb{Q}$ and $\Sigma_{0}$ contains all the primes of bad reduction of $E_{i}, i=1,2$. A similar comparison has been made in [SS, Theorem 3.4] where we study the simultaneous vanishing of imprimitive Selmer groups of congruent elliptic curves under certain stronger conditions on the set $\Sigma_{0}$.

We briefly explain below how the result of Greenberg and Vatsal can be extended over more general number fields and for any set $\Sigma_{0}$ containing primes dividing $N_{K} / \bar{N}_{K}$. Let $\Sigma_{0} \subset \Sigma \backslash p$ be a finite set of primes of $K$ containing the prime divisors of $N_{K} / \bar{N}_{K}$. Consider the commutative diagram

(6)

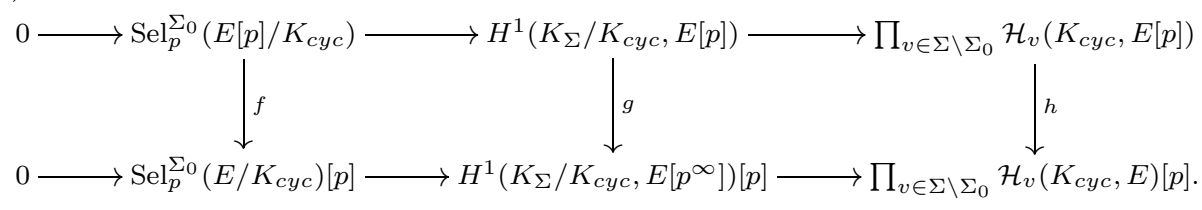

Assumption 1(ii) implies that $E(K)[p]=0$. From the Nakayama lemma we get that $E\left(K_{c y c}\right)[p]=0$. This implies that $H^{0}\left(K_{\Sigma} / K_{c y c}, E\left[p^{\infty}\right]\right) / p=0$ and therefore $g$ is injective. From Lemma $2.1 h$ is injective. Now an application of the snake lemma implies that the map

$$
f: \operatorname{Sel}_{p}^{\Sigma_{0}}\left(E[p] / K_{c y c}\right) \longrightarrow \operatorname{Sel}_{p}^{\Sigma_{0}}\left(E / K_{c y c}\right)[p]
$$

is an isomorphism. Therefore, $\operatorname{Sel}_{p}^{\Sigma_{0}}\left(E / K_{c y c}\right)[p]$ is finite if and only if $\operatorname{Sel}_{p}^{\Sigma_{0}}\left(E[p] / K_{c y c}\right)$ is finite and

$$
\lambda^{\Sigma_{0}}=\operatorname{rank}_{\mathbb{Z} / p} \operatorname{Sel}_{p}^{\Sigma_{0}}\left(E[p] / K_{c y c}\right) .
$$

We mention that here we have used the fact that the Pontryagin dual of $\operatorname{Sel}_{p}^{\Sigma_{0}}\left(E / K_{c y c}\right)[p]$ is isomorphic to $X^{\Sigma_{0}}\left(E / K_{c y c}\right) / p$.

Let $E_{1}$ and $E_{2}$ be two elliptic curves defined over the field of rational numbers $\mathbb{Q}$ and let $\tilde{E}_{i}$ denote the reduced curve at $p$ for $i=1,2$. Suppose that $E_{1}[p] \cong E_{2}[p]$ as $\operatorname{Gal}(\overline{\mathbb{Q}} / \mathbb{Q})$-modules. Let $\Sigma$ be the set of primes of $K$ containing the primes of bad reduction of $E_{1}$ and $E_{2}$, the primes above $p$ and infinite primes. Now consider the set

$$
\Sigma_{0}:=\left\{v \in \Sigma|v| N_{1} / \bar{N}_{1} \text { or } v \mid N_{2} / \bar{N}_{2}\right\}
$$

as defined in (2). Suppose that $E_{1}[p] \cong E_{2}[p]$ as $\operatorname{Gal}(\overline{\mathbb{Q}} / \mathbb{Q})$-modules. Since $E_{i}$ has good ordinary reduction at $p, \tilde{E}_{i, p}[p]$ is characterised as the maximal unramified quotient of $E_{i}[p]$, for $i=1,2$ (see [Gr1, Section 2]). In particular, the $\operatorname{Gal}(\overline{\mathbb{Q}} / \mathbb{Q})$ module isomorphism $E_{1}[p] \cong E_{2}[p]$ induces the isomorphism $\tilde{E}_{1, p}[p] \cong \tilde{E}_{2, p}[p]$ of $\operatorname{Gal}\left(\overline{\mathbb{Q}}_{p} / \mathbb{Q}_{p}\right)$-modules. Therefore we have an isomorphism $\tilde{E}_{1, p}[p] \cong \tilde{E}_{2, p}[p]$ of $\operatorname{Gal}\left(\bar{K}_{v} / K_{v}\right)$-modules via the restriction of the Galois action. Since $E_{1}$ and $E_{2}$ have 
good ordinary reduction at $p$, both $E_{1}$ and $E_{2}$ have good ordinary reduction at every prime $v \mid p$ of $K$ and $\tilde{E}_{i, v}[p]=\tilde{E}_{i, p}[p]$ for $i=1,2$. Therefore, $\tilde{E}_{1, v}[p] \cong \tilde{E}_{2, v}[p]$ as $\operatorname{Gal}\left(\bar{K}_{v} / K_{v}\right)$-modules for every prime $v \mid p$ of $K$. This implies that

$$
\operatorname{Sel}_{p}^{\Sigma_{0}}\left(E_{1}[p] / K_{c y c}\right) \cong \operatorname{Sel}_{p}^{\Sigma_{0}}\left(E_{2}[p] / K_{c y c}\right) .
$$

From equation (8) and the isomorphisms (77) and (10) we get the following

Theorem 2.2. Suppose that Assumption 1 (i), (ii) and (iii) hold and let $\Sigma_{0}$ be the finite set of primes of $K$ as defined in (2). Then the assumption 1 (iv) holds for $E_{1}$ if and only if it holds for $E_{2}$. Further, if the assumption 1 (iv) holds for $E_{1}$, then $\lambda_{E_{1}}^{\Sigma_{0}}=\lambda_{E_{2}}^{\Sigma_{0}}$.

\section{Parity of $\lambda$-invariants of CONGRUEnt Elliptic CURVE}

Let $v \nmid p$ be a finite prime of $K$. Then for every prime $w \mid v$ of $K_{c y c}$ and an elliptic curve $E$ defined over $K$, the Pontryagin dual of $H^{1}\left(K_{c y c, w}, E\left[p^{\infty}\right]\right)$ is a finitely generated $\mathbb{Z}_{p}$-module (see for example Gr2, Proposition 2]). Let $\sigma_{E}^{w}$ denote the $\mathbb{Z}_{p^{-}}$ corank of $H^{1}\left(K_{c y c, w}, E\left[p^{\infty}\right]\right)$. Using the fact that there are finitely many primes of $K_{c y c}$ above $v$, we get that the Pontryagin dual of $\mathcal{H}_{v}\left(K_{c y c}, E\right)$ is a finitely generated $\mathbb{Z}_{p}$-module. Let $\tau_{E}^{v}$ denote the $\mathbb{Z}_{p}$-rank of the Pontryagin dual of $\mathcal{H}_{v}\left(K_{c y c}, E\right)$. By an argument similar to [Gr1, Page 237] (using the exact sequence (5)) we have the following

Lemma 3.1. Suppose that Assumption 1(i) and 1(iv) hold. Let $\Sigma_{0}$ be a finite set of finite primes of $K$ not containing the primes above $p$. Then $\lambda_{E_{i}}^{\Sigma_{0}}=\lambda_{E_{i}}+\Sigma_{v \in \Sigma_{0}} \tau_{E_{i}}^{v}$ for $i=1,2$.

We mention that as in Gr1] to prove the above lemma, the assumption that the Pontryagin dual of $\operatorname{Sel}\left(E / K_{c y c}\right)$ is a torsion $\Lambda$-module is needed. The Assumption 1 (iv) implies that the Pontryagin dual of $\operatorname{Sel}\left(E / K_{c y c}\right)$ is a finitely generated $\mathbb{Z}_{p^{-}}$ module and therefore it is also a torsion $\Lambda$-module.

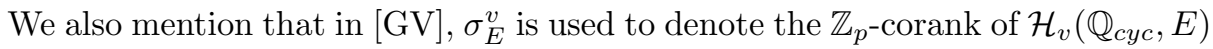
for every prime $v$ of $\mathbb{Q}$ not equal to $p$. Whereas, we use the notation $\tau_{E}^{v}$ to denote the $\mathbb{Z}_{p}$-corank of $\mathcal{H}_{v}\left(K_{c y c}, E\right)$ for every prime $v$ of the number field $K$ not dividing $p$ and $\sigma_{E}^{w}$ to denote the $\mathbb{Z}_{p}$-corank of $H^{1}\left(K_{c y c, w}, E\left[p^{\infty}\right]\right)$ for every prime $w \mid v$ of $K_{c y c}$. Next, we show that if $v \nmid p$ is a finite prime of $K$ and $w \mid v$ and $w^{\prime} \mid v$ are primes of $K_{c y c}$, then $\sigma_{E}^{w}=\sigma_{E}^{w^{\prime}}$.

For every finite prime $v$ of $K$, let $s_{v}$ denote the number of primes of $K_{c y c}$ lying above $v$. Note that $s_{v}$ is a power of $p$. In particular $s_{v}$ is odd. Let $w$ and $w^{\prime}$ be two primes of $K_{c y c}$ lying above $v$ and let $\eta \in \Gamma=\operatorname{Gal}\left(K_{c y c} / K\right)$ such that $\eta(w)=w^{\prime}$. Then $\eta$ induces an isomorphism between $H^{1}\left(K_{c y c, w}, E\left[p^{\infty}\right]\right)$ and $H^{1}\left(K_{c y c, w^{\prime}}, E\left[p^{\infty}\right]\right)$. Therefore $\sigma_{E}^{w}=\sigma_{E}^{w^{\prime}}$. This implies that $\tau_{E}^{v}=s_{v} \sigma_{E}^{w}$ for every prime $w \mid p$. Since $s_{v}$ is odd we have the following

Lemma 3.2. Let $v \nmid p$ be a finite prime of $K$ and let $w \mid v$ be a prime of $K_{c y c}$. Then we have that $\tau_{E}^{v}=\sigma_{E}^{w} \bmod 2$.

Lemma 3.3. Let $v \nmid p$ be a finite prime of $K$ and $w \mid v$ be a prime of $K_{c y c}$. If Assumption 1(iii) holds and $E$ has split multiplicative reduction at $v$, then

$$
H^{1}\left(K_{c y c, w}, E\left[p^{\infty}\right]\right)=\mathbb{Q}_{p} / \mathbb{Z}_{p} .
$$

In particular, if $E$ has split multiplicative reduction at $v$, then $\sigma_{E}^{w}=1$. 
Proof. Let $J$ be the extension $K_{v}\left(E\left[p^{\infty}\right]\right)$ of $K_{v}$ obtained by adjoining the coordinates of points in $E\left[p^{\infty}\right]$. We have that $H^{1}\left(J, E\left[p^{\infty}\right]\right)=0$ (see OV, Lemma 5.4(ii)] and [CH, Lemma 5.1(ii)]). From the Hochschild-Serre spectral sequence we get that $H^{1}\left(\operatorname{Gal}\left(J / K_{c y c, w}\right), E\left[p^{\infty}\right]\right) \cong H^{1}\left(K_{c y c, w}, E\left[p^{\infty}\right]\right)$. Now, the lemma follows from [CH, Lemma 5.13(i)].

Remark 3.4. Consider the elliptic curve

$$
E: y^{2}=x^{3}-584 x+5444
$$

with conductor 364 . The curve $E$ has good and ordinary reduction at the prime $p=5$ and split multiplicative reduction at 7 . It is shown in GV, Page 44] that for every prime $w \mid 7$ of $\mathbb{Q}_{c y c}, \sigma_{E}^{w}=0$. This shows that the Assumption 1(iii) is necessary in the above lemma.

Lemma 3.5. Let $v \nmid p$ be a finite prime of $K$ and $w \mid v$ be a prime of $K_{c y c}$. Suppose that $E$ has either additive reduction at $v$ or non-split, multiplicative reduction at $v$. If Assumption 1 (iii) holds, then $H^{1}\left(K_{c y c, w}, E\left[p^{\infty}\right]\right)=0$. In particular, if $E$ has non-split, multiplicative reduction at $v$ or additive reduction at $v$, then $\sigma_{E}^{w}=0$.

Proof. Let $T_{p} E$ denote the Tate module of $E$ and put $E^{\star}\left[p^{\infty}\right]:=\operatorname{Hom}_{\mathbb{Z}_{p}}\left(T_{p} E, \mu_{p}\right)$. Then from [Gr2, Proposition 2], we have that $H^{1}\left(K_{c y c, w}, E\left[p^{\infty}\right]\right)$ is pseudoisomorphic to $H^{0}\left(K_{c y c, w}, E^{\star}\left[p^{\infty}\right]\right)$. Since $E$ is self dual, $E^{\star}\left[p^{\infty}\right] \cong E\left[p^{\infty}\right]$. Therefore, $H^{0}\left(K_{c y c, w}, E^{\star}\left[p^{\infty}\right]\right) \cong H^{0}\left(K_{c y c, w}, E\left[p^{\infty}\right]\right)$. Now, using the assumption of the lemma and [HM, Proposition 5.1] we get that $H^{0}\left(K_{c y c, w}, E\left[p^{\infty}\right]\right)$ is finite. Therefore $H^{1}\left(K_{c y c, w}, E\left[p^{\infty}\right]\right)$ is finite. Now, it is well known that $H^{1}\left(K_{c y c, w}, E\left[p^{\infty}\right]\right)$ is divisible (see [Gr. Lemma 4.5 and the successive paragraph]). This implies that $H^{1}\left(K_{c y c, w}, E\left[p^{\infty}\right]\right)=0$.

Lemma 3.6. Let $v \nmid p$ be a finite prime of $K$ and $w \mid v$ be a prime of $K_{c y c}$. Suppose that Assumption 1 (iii) holds. If $E$ has good reduction at the prime $v$, then $\sigma_{E}^{w}$ is even.

Proof. First we suppose that $H^{0}\left(K_{v}, E[p]\right)=0$. Then from [HM, Proposition 5.1] we get that $H^{0}\left(K_{c y c, w}, E\left[p^{\infty}\right]\right)=0$. By an argument similar to the proof of Lemma 3.5 we have that $H^{1}\left(K_{c y c, w}, E\left[p^{\infty}\right]\right)=0$. Now suppose that $H^{0}\left(K_{v}, E[p]\right) \neq 0$. From [HM, Proposition 5.1] we get that $H^{0}\left(K_{c y c, w}, E\left[p^{\infty}\right]\right)=E\left[p^{\infty}\right]$. Therefore $H^{1}\left(K_{c y c, w}, E\left[p^{\infty}\right]\right) \cong H^{1}\left(K_{c y c, w},\left(\mathbb{Q}_{p} / \mathbb{Z}_{p}\right)^{2}\right)$. This implies that $\sigma_{E}^{w}$ is even.

Remark 3.7. Consider the elliptic curve

$$
E: y^{2}=x^{3}+x-10
$$

with conductor 52 over $\mathbb{Q}$. The curve $E$ has good and ordinary reduction at $p=5$ and good reduction at the prime 7 . It is shown in [GV, Page 44] that for every prime $w \mid 7$ of $\mathbb{Q}_{c y c}, \sigma_{E}^{w}$ is 1 . This shows that the Assumption 1(iii) is necessary in the above lemma.

Consider a set of finite primes $\Sigma_{0} \subset \Sigma \backslash p$. Let $S_{E_{i}}$ be the set of primes in $\Sigma_{0}$ where $E_{i}$ has split multiplicative reduction for $i=1,2$. Using Lemma 3.1, Lemma 3.3. Lemma 3.5 and Lemma 3.6 we have the following

Lemma 3.8. Suppose that Assumption 1 holds. Let $\Sigma_{0} \subset \Sigma \backslash p$ be a finite set of finite primes of $K$. Then $\lambda_{E_{i}}^{\Sigma_{0}}=\lambda_{E_{i}}+\left|S_{E_{i}}\right| \bmod 2$, where $\left|S_{E_{i}}\right|$ denotes the cardinality of $S_{E_{i}}$ for $i=1,2$. 
As before we consider two elliptic curves $E_{1}$ and $E_{2}$ defined over $\mathbb{Q}$ such that $E_{1}[p] \cong E_{2}[p]$ and consider the set $\Sigma_{0}$ as defined in (2). Let $S_{E_{1}}$ (resp. $S_{E_{2}}$ ) denote the set of primes in $\Sigma_{0}$ where $E_{1}$ (resp. $E_{2}$ ) has split multiplicative reduction.

Theorem 3.9. Suppose that Assumption 1 holds. Let $\Sigma_{0}$ be the finite set of finite primes of $K$ as defined in (2). If $E_{1}[p] \cong E_{2}[p]$ as $\operatorname{Gal}(\overline{\mathbb{Q}} / \mathbb{Q})$-modules, then $\lambda_{E_{1}}+$ $\left|S_{E_{1}}\right|=\lambda_{E_{2}}+\left|S_{E_{2}}\right| \bmod 2$.

Proof. The theorem follows from Theorem 2.2 and Lemma 3.8

\section{Parity of the RANK of CONGRUent Elliptic CURVes}

For an elliptic curve $E$ defined over $K$, let $s_{p}(E / K)$ denote the $\mathbb{Z}_{p}$-rank of the Pontryagin dual of $\operatorname{Sel}_{p}(E / K)$. Let $r(E / K)$ denote the rank of $E$ over $K$. As in the previous sections, let $E_{1}$ and $E_{2}$ be two elliptic curves defined over $\mathbb{Q}$ such that $E_{1}[p] \cong E_{2}[p]$ as $\operatorname{Gal}(\overline{\mathbb{Q}} / \mathbb{Q})$-module. Throughout this section, we shall assume that $\Sigma_{0}$ is the finite set of finite primes of $K$ as defined in (2) and $S_{E_{1}}$ (resp. $S_{E_{2}}$ ) denotes the set of primes in $\Sigma_{0}$ where $E_{1}$ (resp. $E_{2}$ ) has split multiplicative reduction.

Theorem 4.1. Let $E$ be an elliptic curve defined over $K$ and suppose that the Pontryagin dual of $\operatorname{Sel}_{p}\left(E / K_{c y c}\right)$ is $\Lambda$-torsion. Then

$$
s_{p}(E / K) \equiv \lambda_{E} \bmod 2 .
$$

Proof [Gr, Proposition 3.10].

Theorem 4.2. Let $E_{1}$ and $E_{2}$ be two elliptic curves defined over $\mathbb{Q}$ such that $E_{1}[p] \cong E_{2}[p]$ as $\operatorname{Gal}(\overline{\mathbb{Q}} / \mathbb{Q})$-modules and $\Sigma_{0}$ be the finite set of finite primes of $K$ as defined in (2). Suppose that Assumption 1 holds for $E_{1}$ and $E_{2}$. Then we have that

$$
s_{p}\left(E_{1} / K\right)+\left|S_{E_{1}}\right|=s_{p}\left(E_{2} / K\right)+\left|S_{E_{2}}\right| \bmod 2 .
$$

Proof. The theorem follows from Theorem 3.9 and Theorem 4.1 .

For an elliptic curve $E$ defined over the number field $K$, let $L(E / K, s)$ denote the Hasse-Weil $L$-function of $E$ over $K$. Let $E$ be an elliptic curve defined over $\mathbb{Q}$ and $K$ be a solvable Galois extension of $\mathbb{Q}$. It is a consequence of the Modularity theorem proved by Wiles et al. and the theory of base-change for automorphic representations of $G L(n)$ that the Hasse-Weil $L$-function $L(E / K, s)$ has an analytic continuation to the entire complex plane (cf. $[\mathrm{C},[\mathrm{W},[\mathrm{BCDT})$. For a solvable extension $K / \mathbb{Q}$, let $a(E / K)$ denote the order of zero of $L(E / K, s)$ at 1 .

Conjecture 1 (p-parity conjecture). $s_{p}(E / K)=a(E / K) \bmod 2$.

Theorem 4.3 ([DD, Theorem 6.13], [DD, Theorem 6.15], [DD, Example 6.16]). Let $E$ be an elliptic curve defined over $\mathbb{Q}$. The p-parity conjecture holds for $E$ over Galois extension $K / \mathbb{Q}$ if $K \subset F$ where one of the following holds:

(i) $F=\mathbb{Q}\left(\mu_{p^{n}}, m^{1 / p^{n}}\right)$ for $m, n \geq 1$, integer.

(ii) $p \equiv 3 \bmod 4$ and $F$ is a Galois $p$-extension of an abelian extension of $\mathbb{Q}$, Galois over $\mathbb{Q}$.

(iii) $p=3$ and $F \subset \mathbb{Q}\left(E\left[3^{\infty}\right]\right)$.

Corollary 4.4. Let $K \subset F$ be a Galois extension of $\mathbb{Q}$ where one of the following holds:

(i) $F=\mathbb{Q}\left(\mu_{p^{n}}, m^{1 / p^{n}}\right)$ for $m, n \geq 1$, integer. 
(ii) $p \equiv 3 \bmod 4$ and $F$ is a Galois $p$-extension of an abelian extension of $\mathbb{Q}$, Galois over $\mathbb{Q}$.

(iii) $p=3$ and $F \subset \mathbb{Q}\left(E_{1}\left[3^{\infty}\right]\right) \cap \mathbb{Q}\left(E_{2}\left[3^{\infty}\right]\right)$.

Let $\Sigma_{0}$ be the finite set of primes of $K$ as defined in (2). Further suppose that Assumption 1 holds and that $E_{1}[p] \cong E_{2}[p]$ as $\operatorname{Gal}(\overline{\mathbb{Q}} / \mathbb{Q})$-modules. Then,

$$
a\left(E_{1} / K\right)+\left|S_{E_{1}}\right|=a\left(E_{2} / K\right)+\left|S_{E_{2}}\right| \bmod 2 .
$$

Finally, we mention that if the 2-primary and 3-primary subgroup of the TateShafarevich group $\amalg(E / K(E[2]))$ of an elliptic curve $E$ is finite over the field $K(E[2])$ satisfying the assumptions of Theorem 4.3, then it is known that the parity of the rank of $E$ over $K$ is the same as the parity of $s_{p}(E / K)$ (see [DD, Theorem 7.1]). Using this fact, we can also determine the variation of the parity of ranks of elliptic curves under congruence.

We now illustrate our result with an example. Consider the pair of elliptic curves $E_{1}$ and $E_{2}$ defined by

$$
E_{1}=11 A 1: y^{2}+y=x^{3}-x^{2}-10 x-20
$$

and

$$
E_{2}=121 C 1: y^{2}+x y=x^{3}+x^{2}-2 x-7 .
$$

We have that $E_{1}[3] \cong E_{2}[3]$ as $\operatorname{Gal}(\overline{\mathbb{Q}} / \mathbb{Q})$-module. Both the curves satisfy Assumption 1 (i) and (ii) for $K=\mathbb{Q}\left(\mu_{p}\right)$. The curve $11 A 3$ in the Cremona table is 5 -isogenus to the curve 11A1. From [DD1, Table B.1.] it follows that the Iwasawa $\mu$-invariant of $11 A 3$ vanishes over $K_{c y c}$. Since $11 A 3$ does not have any 3 isogeny defined over $K$, the $\mu$-invariant of $11 A 3$ and $11 A 1$ are the same. Therefore Assumption 1 (iv) holds for $E_{1}$. The prime to 3-conductor of $E_{1}[3]$ is 11 over $K$. Therefore $11=N_{2} / \bar{N}_{2}$. Since $E_{1}$ has split multiplicative reduction at $11, S_{E_{1}}=\{11\}$. The curve $E_{2}$ has additive reduction at 11 and therefore $S_{E_{2}}$ is empty. Using Sage we get that the analytic rank of $E_{1}$ is zero over $K$ and the analytic rank of $E_{2}$ is 1 over $K$. Thus, using Theorem 4.3 we get that $s_{3}\left(E_{1} / K\right)+\left|S_{E_{1}}\right|=0+1 \bmod 2$ and $s_{3}\left(E_{2} / K\right)+\left|S_{E_{2}}\right|=1+0 \bmod 2$. In particular, $s_{3}\left(E_{1} / K\right)+\left|S_{E_{1}}\right|=s_{3}\left(E_{2} / K\right)+\left|S_{E_{2}}\right|$ $\bmod 2$.

In Remark 3.4 (resp. Remark 3.7) we found that Lemma 3.3 (resp. Lemma 3.6) fails if $K \neq K\left(\mu_{p}\right)$. Both these lemmas were crucially used in Theorem 1.1. We do not have a counterexample of this theorem when $K \neq K\left(\mu_{p}\right)$. We shall next discuss a few examples which in fact suggest that Theorem 1.1 could still be true without the assumption that $\mu_{p} \subset K$. Consider the pair of elliptic curves $E 1$ and $E 2$, where $E_{1}=235 A 1$ and $E_{2}=235 B 1$ from the Cremona table with Weierstrass equation

$$
E_{1}: y^{2}+x y+y=x^{3}+x^{2}-3551 x-82926
$$

and

$$
E_{2}: y^{2}+x y+y=x^{3}+x^{2}-5 x,
$$

respectively. Then, using Sage we get that $E_{1}[3] \cong E_{2}[3]$ as $\operatorname{Gal}(\overline{\mathbb{Q}} / \mathbb{Q})$-module. Further both $E_{1}$ and $E_{2}$ have good and ordinary reduction at 3 and $E_{1}[3]$ is an irreducible $\operatorname{Gal}(\overline{\mathbb{Q}} / K)$-module. The curve $E_{1}$ has analytic rank 0 whereas the curve $E_{2}$ has analytic rank 1 over $\mathbb{Q}$. The prime to 3 conductor of $E_{i}[3]$ over $\mathbb{Q}$ is 47 and $5=N_{i} / \bar{N}_{i}$ for $i=1,2$. Over $\mathbb{Q}$ the curve $E_{1}$ has non-split multiplicative reduction and $E_{2}$ has split multiplicative reduction at 5 . In particular, $S_{E_{1}}(\mathbb{Q})=0$ 
and $S_{E_{2}}(\mathbb{Q})=1$. Therefore, $a\left(E_{1} / \mathbb{Q}\right)+S_{E_{1}}(\mathbb{Q})=0$ and $a\left(E_{2} / \mathbb{Q}\right)+S_{E_{2}}(\mathbb{Q})=2$. Thus, using Theorem 4.3 we see that the conclusion of Theorem 1.1

$$
s_{3}\left(E_{1} / \mathbb{Q}\right)+S_{E_{1}}(\mathbb{Q})=s_{3}\left(E_{2} / \mathbb{Q}\right)+S_{E_{2}}(\mathbb{Q}) \bmod 2
$$

holds over $\mathbb{Q}$. Both curves have split multiplicative reduction at 5 over $K=\mathbb{Q}\left(\mu_{3}\right)$. In particular, $\left|S_{E_{1}}\right|=\left|S_{E_{2}}\right|=1$ over $K$. Using Sage, we also get that both curves have analytic rank 1 over $K$. Thus, using Theorem 4.3 we see that the conclusion of Theorem 1.1

$$
s_{p}\left(E_{1} / K\right)+\left|S_{E_{1}}\right|=s_{p}\left(E_{2} / K\right)+\left|S_{E_{2}}\right| \bmod 2
$$

also holds over $K$. The elliptic curves

$$
E_{1}: y^{2}=x^{3}+x-10
$$

and

$$
E_{2}: y^{2}=x^{3}-584 x+5444
$$

in Remarks 3.4 and 3.7 are congruent mod 5 (see [GV]). The curve $E_{1}$ has conductor 52 and $E_{2}$ has conductor 364. Both these curves satisfy the Assumptions 1 (i), (ii) and (iv). The prime to 5 conductor of $E_{1}[5]$ is equal to $52, \Sigma_{0}$ contains 7 and $E_{2}$ has split multiplicative reduction at 7 . Therefore, $\left|S_{2}(\mathbb{Q})\right|=1$ and $\left|S_{1}(K)\right|=0$. The analytic rank of $E_{1}$ over $\mathbb{Q}$ is 0 and the analytic rank of $E_{2}$ over $\mathbb{Q}$ is 1 . From this we get that the conclusion of Theorem 1.1 holds for $E_{1}$ and $E_{2}$. Further, it can be easily verified that the elliptic curves $E_{1}$ and $E_{2}$ satisfy the relation (3) over $\mathbb{Q}\left(\mu_{5}\right)$.

Next we discuss some examples of a family of elliptic curves that are congruent at the prime $p$ and explain how our result can be used to determine the parity of their ranks.

Theorem 4.5 ([RS, Theorem 4.1]). Fix an elliptic curve E over $\mathbb{Q}$,

$$
y^{2}=x^{3}+a x+b,
$$

and let $J=4 a^{3} /\left(4 a^{3}+27 b^{2}\right)$. Define

$$
\begin{gathered}
a(t)=\left(\left(-11 J^{2}+28 J-16\right) t^{4}-4\left(2 J^{2}-7 J+4\right) t^{3}+6 J t^{2}+4 t+1\right) a, \\
b(t)=\left(\left(-8 J^{4}+35 J^{3}-4 J^{2}-80 J+64\right) t^{6}+12\left(J^{3}+6 J^{2}-20 J+16\right) t^{5}\right. \\
\left.+15\left(5 J^{2}-16 J+16\right) t^{4}+20\left(J^{2}-5 J+8\right) t^{3}-15(J-4) t^{2}+12 t+1\right) b
\end{gathered}
$$

and define $E_{t}$ by

$$
y^{2}=x^{3}+a(t) x+b(t) .
$$

Then for every $t \in \mathbb{Q}$ such that $E_{t}$ is non-singular, $E_{t}[3]$ is isomorphic as a $\operatorname{Gal}(\overline{\mathbb{Q}} / \mathbb{Q})$-module to $E[3]$.

We consider the elliptic curve $E$ of conductor 11 defined by the equation

$$
y^{2}=x^{3}-432 x+8208 .
$$

The curve $E$ has good and ordinary reduction at the prime 3 . This implies that $E[3]$ is a reducible $\operatorname{Gal}\left(\overline{\mathbb{Q}}_{3} / \mathbb{Q}_{3}\right)$-module and therefore every elliptic curve congruent mod 3 to $E$ has ordinary reduction at 3 . From [DD1, Table 3-11A3] we get that $s_{3}(E / K)=a(E / K)=0$ for $K=\mathbb{Q}\left(\mu_{3}\right)$. The $\operatorname{Gal}(\bar{K} / K)$-module $E[3]$ is irreducible and the conductor of $E$ is equal to the prime to 3 -conductor $\bar{N}$ of $E[3]$ (see SS, Section 5]). From [DD, Table B.1.] we get that Assumption 1(iv) holds. If the conductor of $E_{t}$ is square free at 11 , then $11 \notin \Sigma_{0}$ and $S_{E}$ is empty. In this case 
$S_{E_{t}}$ denotes the set of primes $v \nmid \bar{N}$ of $K$ where $E_{t}$ has split multiplicative reduction. Theorem 4.2 and Corollary 4.4 implies that

$$
s_{3}\left(E_{t} / \mathbb{Q}\left(\mu_{3}\right)\right)=a\left(E_{t} / \mathbb{Q}\left(\mu_{3}\right)\right) \equiv\left|S_{E_{t}}\right| \bmod 2 .
$$

If the conductor of $E_{t}$ is not square free, then $\Sigma_{0}$ contains 11. In this case $\left|S_{E}\right|=1$ and we have

$$
s_{3}\left(E_{t} / \mathbb{Q}\left(\mu_{3}\right)\right)=a\left(E_{t} / \mathbb{Q}\left(\mu_{3}\right)\right) \equiv\left|S_{E_{t}}\right|+1 \bmod 2 .
$$

Note that since $E$ has good reduction at 3 , there are infinitely many rational numbers $t$ such that $E_{t}$ has good reduction at 3 .

Next consider the elliptic curve $\mathrm{E}=158 \mathrm{C} 1$ from the Cremona table. The residual representation at $p=3$ is again irreducible with conductor equal to 158 . It can be easily checked using Sage that the curve $E$ satisfies the Assumption 1 (i) and (ii) over $K=\mathbb{Q}\left(\mu_{3}\right)$. Further the 3 -adic $L$-value of $E$ is a 3-adic unit over $K$ (see [SS, Section 5] for the precise statement). Using this fact and the Iwasawa main conjecture of elliptic curves over cyclotomic $\mathbb{Z}_{p}$-extensions, it can be shown that the $p$-Selmer group of $E$ over $K_{c y c}$ vanishes. In particular, the Assumption 1(iv) holds. The rank of $E$ is zero over $K$ and using Theorem 4.5 we get a family of elliptic curves $E_{t}$ such that $E_{t}[3] \cong E[3]$ as $\operatorname{Gal}(\overline{\mathbb{Q}} / \mathbb{Q})$-module. The curve $E$ has split multiplicative reduction at 2 and non-split multiplicative reduction at the prime factors of $79 \mathrm{in} K$. If the conductor of $E_{t}$ is square free at 2 , then $2 \notin \Sigma_{0}$. Thus, once again we have that

$$
s_{3}\left(E_{t} / \mathbb{Q}\left(\mu_{3}\right)\right)=a\left(E_{t} / \mathbb{Q}\left(\mu_{3}\right)\right) \equiv\left|S_{E_{t}}\right| \bmod 2 .
$$

If the conductor of $E_{t}$ is not square free at 2 , then $2 \in \Sigma_{0}$. Therefore $\left|S_{E}\right|=1$. Therefore as in the previous example we have

$$
s_{3}\left(E_{t} / \mathbb{Q}\left(\mu_{3}\right)\right)=a\left(E_{t} / \mathbb{Q}\left(\mu_{3}\right)\right) \equiv\left|S_{E_{t}}\right|+1 \bmod 2 .
$$

Given an elliptic curve $E$ defined over $\mathbb{Q}$, Rubin and Silverberg also produce a family of elliptic curves $E_{t}$ where $t \in \mathbb{Q}$, such that $E_{t}[5] \cong E[5]$ for every $t$ (see $[\mathrm{RS}$, Theorem 5.1] for the precise statement). We can also compute the parity of the ranks of elliptic curves in such families using our result.

\section{ACKNOWLEDGEMENT}

We would like to thank Professor R. Sujatha for numerous discussions and helpful comments. This work was written when the author was a Postdoctoral fellow at the Mathematics Center Heidelberg (MATCH) and he would like to thank Professor Otmar Venjakob for the help and support during his stay in Heidelberg. We thank the anonymous referee for sparing valuable time in reading the article very carefully and highly appreciate the comments and suggestions, which significantly contributed to improving the quality of the article. Finally, we thank the Mathematics Center Heidelberg and DST, Government of India for their support during the preparation of this article.

\section{REFERENCES}

[BCDT] Christophe Breuil, Brian Conrad, Fred Diamond, and Richard Taylor, On the modularity of elliptic curves over Q: wild 3-adic exercises, J. Amer. Math. Soc. 14 (2001), no. 4, 843-939 (electronic), DOI 10.1090/S0894-0347-01-00370-8. MR.1839918(2002d:11058) 
[C] L. Clozel, Base change for GL(n), Proceedings of the International Congress of Mathematicians, Vol. 1, 2 (Berkeley, Calif., 1986), Amer. Math. Soc., Providence, RI, 1987, pp. 791-797. MR934282(89i:22034)

[Dr] Michael Jeffrey Drinen, Iwasawa mu-invariants of Selmer groups, ProQuest LLC, Ann Arbor, MI, 1999. Thesis (Ph.D.)-University of Washington. MR2699373

[Dr1] Michael J. Drinen, Finite submodules and Iwasawa $\mu$-invariants, J. Number Theory 93 (2002), no. 1, 1-22, DOI 10.1006/jnth.2001.2717. MR.1892927 (2003b:11118)

[Gr] Ralph Greenberg, Iwasawa theory for elliptic curves, Arithmetic theory of elliptic curves (Cetraro, 1997), Lecture Notes in Math., vol. 1716, Springer, Berlin, 1999, pp. 51-144, DOI 10.1007/BFb0093453. MR 1754686(2002a:11056)

[Gr1] Ralph Greenberg, Selmer groups and congruences, Proceedings of the International Congress of Mathematicians. Volume II, Hindustan Book Agency, New Delhi, 2010, pp. 231248. MR2827793 (2012j:11119)

[Gr2] Ralph Greenberg, Iwasawa theory for p-adic representations, Algebraic number theory, Adv. Stud. Pure Math., vol. 17, Academic Press, Boston, MA, 1989, pp. 97-137. MR:1097613 (92c:11116)

[GV] Ralph Greenberg and Vinayak Vatsal, On the Iwasawa invariants of elliptic curves, Invent. Math. 142 (2000), no. 1, 17-63, DOI 10.1007/s002220000080. MR1784796 (2001g:11169)

[EPW] Matthew Emerton, Robert Pollack, and Tom Weston, Variation of Iwasawa invariants in Hida families, Invent. Math. 163 (2006), no. 3, 523-580, DOI 10.1007/s00222-0050467-7. MR2207234 (2007a:11059)

[CH] J. H. Coates and S. Howson, Euler characteristics and elliptic curves. II, J. Math. Soc. Japan 53 (2001), no. 1, 175-235, DOI 10.2969/jmsj/05310175. MR 1800527 (2001k:11215)

[HM] Yoshitaka Hachimori and Kazuo Matsuno, An analogue of Kida's formula for the Selmer groups of elliptic curves, J. Algebraic Geom. 8 (1999), no. 3, 581-601. MR1689359 (2000c:11086)

[HV] Yoshitaka Hachimori and Otmar Venjakob, Completely faithful Selmer groups over Kummer extensions, Doc. Math. Extra Vol. (2003), 443-478 (electronic). Kazuya Kato's fiftieth birthday. MR2046605 (2005b:11072)

[OV] Yoshihiro Ochi and Otmar Venjakob, On the structure of Selmer groups over p-adic Lie extensions, J. Algebraic Geom. 11 (2002), no. 3, 547-580, DOI 10.1090/S1056-3911-0200297-7. MR:1894938 (2003m:11082)

[DD] Tim Dokchitser and Vladimir Dokchitser, Root numbers and parity of ranks of elliptic curves, J. Reine Angew. Math. 658 (2011), 39-64, DOI 10.1515/CRELLE.2011.060. MR:2831512(2012h:11084)

[DD1] T. Dokchitser and V. Dokchitser, Computations in non-commutative Iwasawa theory, Proc. Lond. Math. Soc. (3) 94 (2007), no. 1, 211-272, DOI 10.1112/plms/pdl014. With an appendix by J. Coates and R. Sujatha. MR2294995 (2008g:11106)

[RS] K. Rubin and A. Silverberg, Families of elliptic curves with constant mod p representations, Elliptic curves, modular forms, \& Fermat's last theorem (Hong Kong, 1993), Ser. Number Theory, I, Int. Press, Cambridge, MA, 1995, pp. 148-161. MR1363500 (96j:11078)

[W] Andrew Wiles, Modular elliptic curves and Fermat's last theorem, Ann. of Math. (2) 141 (1995), no. 3, 443-551, DOI 10.2307/2118559. MR1333035 (96d:11071)

[SS] Sudhanshu Shekhar and R. Sujatha, Euler characteristic and congruences of elliptic curves, Münster J. Math. 7 (2014), 327-343. MR3271248

Mathematics Center Heidelberg - And - Indian Institute of Science education And Research, Mohali

E-mail address: sudhanshu@mathi.uni-heidelberg.de

E-mail address: sshekhars2012@gmail.com 